\title{
Clinical Factors to Predict the Response to Concurrent Chemoradiotherapy and Survival in Esophageal Cancer Patients
}

\author{
Su Youn Nam ${ }^{1,2}$, Seong Woo Jeon ${ }^{1,2}$, Sang Jik Lee ${ }^{1}$, Yong Hwan Kwon ${ }^{1,2}$, Hyun Seok Lee ${ }^{1,2}$, and Sung Kook Kim ${ }^{1,2}$ \\ ${ }^{1}$ Department of Internal Medicine, Kyungpook National University Hospital, and ${ }^{2}$ Department of Internal Medicine, School of Medicine, \\ Kyungpook National University, Daegu, Korea
}

Background/Aims: Several clinical factors have been used to predict the response for concurrent chemoradiotherapy (CCRT); however, these factors are insufficient for prognostic predictions. We investigated clinical factors to assess whether they could be used to predict the response to CCRT and the survival of patients with esophageal cancer. Methods: Patients with esophageal cancer underwent CCRT from January 2005 to December 2015. Response to CCRT was classified as progressive disease (PD), stationary disease (SD), partial remission (PR), or complete remission (CR). Factors to predict the response to CCRT and patient survival were subsequently investigated. Results: A total of 535 esophageal cancer patients underwent CCRT. Four hundred ninety-three patients were followed up, and patient outcomes were investigated. In the adjusted analysis, patients with advanced stage disease (relative risk [RR], 0.28 in stage III and 0.12 in stage IV compared to stage I), poor performance status, circumferential involvement (RR, 0.61), and male sex (RR, 0.31 ) were less likely to achieve CR. Advanced stage disease (hazard ratio [HR], 1.71 in stage III/IV), poor CCRT response (HR, 2.82 in PR, 4.47 in SD, 4.77 in PD compared to $\mathrm{CR}$ ), and poor performance status (HR, 1.38 in ECOG 2-4) were found to increase mortality. Conclusions: Advanced stage disease, poor performance status, male sex, and circumferential involvement were independent predictive factors for a poor response to CCRT. Advanced stage, poor performance status, and poor CCRT response were independent factors for decreased survival. (Gut Liver 2020;14:450-458)

Key Words: Esophageal neoplasms; Chemoradiotherapy; Treatment response; Survival

\section{INTRODUCTION}

Esophageal carcinoma is the 7th common malignancy and is the 6th common cause of death in the world. ${ }^{1}$ The incidence of esophageal squamous cell carcinoma (ESCC) is high in China and low in Europe and North America, whereas esophageal adenocarcinoma is more prevalent in Europe and North America. ${ }^{3}$

Esophagectomy is the primary therapeutic modality in early esophageal cancer. However, a lot of patients with esophageal cancer are diagnosed in the advanced stages, with just $20 \%$ of esophageal cancer being resectable. ${ }^{3}$ Concurrent chemoradiotherapy (CCRT) seems to be superior to radiotherapy alone in patients with locally advanced esophageal cancer. ${ }^{4}$ Definitive CCRT is an established management for esophageal cancer, but part of patients achieve initial remission with CCRT alone. Previous studies suggested that tumor extent, lymph node metastasis, and distant metastasis predicted the patient response to CCRT. $^{5-7}$ Some clinical factors have been used for the prediction of CCRT response but those are not highly predictive.

Even if esophagectomy is the first choice of treatment in stage I esophageal cancer, CCRT can be alternative therapy. Patients who have upper esophageal cancer, who are contraindicated for surgery because of comorbidities or who refuse surgery, may be treated with CCRT. In locally advanced esophageal cancer, the overall outcomes of CCRT seems to be comparable to that of surgery. ${ }^{8,9}$ In patients with locally advanced esophageal cancer who respond to CCRT, adjuvant surgery after CCRT has no survival gain compared to CCRT alone. ${ }^{8,9}$ Therefore, predictive factors for CCRT response in all stages of esophageal cancer need to be investigated. Additionally, the ultimate goal of therapy is survival gain. Therefore, we investigated the pretreatment clinical factors for predicting the response of CCRT and survival outcome in patients with esophageal cancer.

\section{Correspondence to: Seong Woo Jeon}

Department of Internal Medicine, Kyungpook National University Hospital, 807 Hoguk-ro, Buk-gu, Daegu 41404, Korea

Tel: +82-53-200-3081, Fax: +82-53-200-3089, E-mail: swjeon@knu.ac.kr

Received on May 14, 2019. Revised on July 29, 2019. Accepted on August 9, 2019. Published online February 3, 2020.

pISSN 1976-2283 eISSN 2005-1212 https://doi.org/10.5009/gnl19165

@ (This is an Open Access article distributed under the terms of the Creative Commons Attribution Non-Commercial License (http://creativecommons.org/licenses/by-nc/4.0) which permits unrestricted non-commercial use, distribution, and reproduction in any medium, provided the original work is properly cited. 


\section{MATERIALS AND METHODS}

\section{Study population and design}

This is a retrospective cohort study from electrical medical records. We searched the patients with codes of International Classification of Disease, 10th revision for esophageal cancer (C15) from January 2005 to December 2015. Patients who only underwent CCRT for esophageal carcinoma at baseline were enrolled and were followed up until July 2017 (Fig. 1). Patients with previous esophageal cancer, those who had synchronous double primary cancer $(n=59)$, and those who received surgery or adjuvant radiotherapy or chemotherapy $(n=129)$, chemotherapy only $(n=71)$, radiotherapy only $(n=37)$, endoscopic resection $(n=38)$, or supportive care $(n=74)$ were excluded. We also excluded those with unknown treatment modalities; referred to other hospital ( $n=119)$, lost to follow-up ( $n=45)$, and those with no available electrical medical data $(n=43)$.

Demographic and clinical findings included age at diagnosis, birth date, sex, underlying chronic diseases (diabetes, hypertension, cardiovascular disease, cerebrovascular disease, chronic renal disease, and chronic liver disease), other cancers (history of other cancer with complete remission), smoking status, drinking status, and performance status. Smoking and drinking status were classified as current, past, and never. Performance status before CCRT was evaluated using the Eastern Cooperative Oncology Group (ECOG) performance status system (score: 0 to 4). This study is a retrospective study using medical record review and so informed consent was waived. This study was approved by the Institutional Review Board of the Kyungpook National University Medical Center (IRB number: KNUMC-2016-05-013).

\section{Tumor characteristics and clinical staging}

Tumor location was defined as cervical $(15$ to $20 \mathrm{~cm}$ from the upper incisor [UI]), upper thorax (20 to $24 \mathrm{~cm}$ from the
UI), mid thorax (24 to $32 \mathrm{~cm}$ from the UI), and lower thoracic esophagus (32 to $40 \mathrm{~cm}$ from the UI). The length was measured using endoscopy, esophageal computed tomography (CT), and positron emission tomography-CT. The tumor shape was classified as protruding, ulcerative, combined, and superficial lesion. Superficial type refers superficial nodular lesion or shallow erosion without ulcer or protruding mass. Protruding type refers to protruding mass without definite ulcer. Ulcerative type refers to ulcer without protruding mass. Combined type refers to protruding mass with definite ulcer. Lumen involvement was classified as partial and circumferential. Initial clinical stage was evaluated using endoscopy, bronchoscopy, esophageal CT, positron emission tomography-CT, and laryngoscopy and was classified based on the American Joint Committee on Cancer Staging Manual.

\section{CCRT regimen and evaluation of CCRT response}

Most patients received 5 days of 5-fluorouracil and cisplatin regimen and radiotherapy. After simulation for radiotherapy, intensity-modulated radiation therapy with 50 to 60 Gy (25 to 30 fractions) of radiation was started along with the first dose of chemotherapy on the local thorax every weekday (radiotherapy for 5 days a week and rest for the weekend). Therefore, it takes 5 to 6 weeks to complete radiotherapy. The first cycle of chemotherapy starts concomitantly with radiotherapy. Chemotherapy protocol is 5 days 5 -fluorouracil and cisplatin and 3 weeks of chemotherapy free period. Therefore, chemotherapy cycle is about 4 weeks interval. The CCRT dose was reduced in those with poor ECOG status and in elderly patients. Small numbers of patients received capecitabine and cisplatin and concurrent radiotherapy.

Treatment response was evaluated using endoscopy with biopsy and esophageal CT at 4 weeks after start of the 2nd, 4th and 6th cycles of chemotherapy. Final clinical response

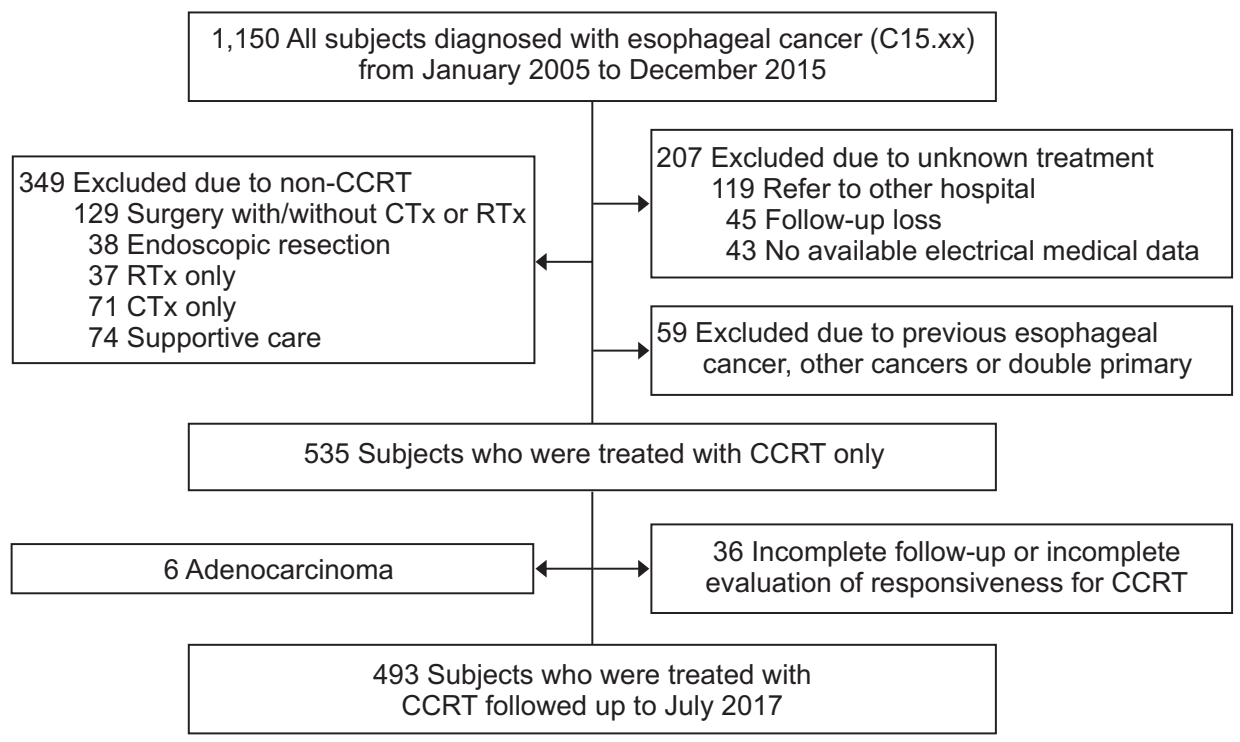

Fig. 1. Study flowchart. Among the 535 subjects who underwent concurrent chemoradiotherapy for esophageal cancer, 493 completed follow-up and were evaluated for concurrent chemoradiotherapy (CCRT) response until July 2017. CTx, chemotherapy; RTx, radiotherapy. 
was evaluated at 4 weeks after 4 to 6 cycles of chemotherapy. Complete remission (CR) was defined as no gross tumor on endoscopy, no pathologic cancer in follow-up endoscopic biopsy, and complete regression of primary tumor, lymph node, and metastatic tumor on radiologic study. Partial remission (PR) was defined as regression of gross tumor on endoscopy or partial regression of primary tumor, lymph node, and metastatic tumor on radiologic study. Stationary disease (SD) was defined as no significant change of gross tumor on endoscopy or no significant change of primary tumor, lymph node, and metastatic tumor on radiologic study. Progressive disease (PD) was defined as disease progression on endoscopic or radiologic study.

\section{Evaluation of survival}

Survival was evaluated using clinical records and change of insurance status from the National Health Insurance System until July 19, 2017.

\section{Statistical analysis}

We used Pearson chi-square test or analysis of variance (ANOVA) to assess the differences among CCRT responses (CR,
PR, SD, and PD) for demographic data (age, sex, chronic disease, other cancers, smoking status, drinking status, and performance status) and tumor characteristics (location, shape, pathology, and clinical stage). Predictive factors for CCRT response were estimated using relative risk (RR) and 95\% confidence interval (CI). Significant variables in the unadjusted analysis were included in the multivariate analysis. We evaluated the ratio of CR compared to that of PR, SD, and PD.

We measured the mean survival by demographic findings, tumor characteristics, and CCRT responses using t-test or ANOVA. Pearson chi-square was used to assess the differences between death and survival according to demographic characteristics, CCRT response, and tumor characteristics. Survival by potentia contributing factors was evaluated with Kaplan-Meier curve, and the risk of death was estimated with hazard ratio (HR) and 95\% CI using Cox regression analysis.

We used STATA version 12 (StataCorp LLC, College Station, TX, USA) for all analyses. All statistical tests were two sided, and p-values less than 0.05 were considered statistically significant.

Table 1. Demographic and Clinical Characteristics According to CCRT Response

\begin{tabular}{|c|c|c|c|c|c|}
\hline Characteristic & CR $(n=221)$ & PR (n=104) & $\mathrm{SD}(\mathrm{n}=50)$ & $\mathrm{PD}(\mathrm{n}=118)$ & p-value ${ }^{*}$ \\
\hline Age, yr & $66.0 \pm 8.5$ & $65.3 \pm 8.1$ & $66.5 \pm 8.4$ & $65.2 \pm 8.9$ & 0.86 \\
\hline Male sex & 206 (93.2) & $100(96.2)$ & 49 (98.0) & 117 (99.2) & 0.06 \\
\hline ECOG & & & & & $<0.001$ \\
\hline 0 & $84(38.7)$ & $29(28.4)$ & $10(20.0)$ & 16 (13.8) & \\
\hline 1 & $102(47.0)$ & $53(52.0)$ & $26(52.0)$ & 66 (56.9) & \\
\hline 2 & $30(13.8)$ & 16 (15.7) & $12(24.0)$ & 25 (21.6) & \\
\hline 3 or 4 & $1(0.5)$ & $4(3.9)$ & $2(3.9)$ & $9(7.7)$ & \\
\hline Chronic disease & & & & & 0.79 \\
\hline Absence & $102(46.2)$ & $52(50.0)$ & $24(48.0)$ & 61 (51.7) & \\
\hline Presence & $119(53.8)$ & $52(50.0)$ & $26(52.0)$ & 57 (48.3) & \\
\hline Other cancers & & & & & 0.99 \\
\hline Absence & 181 (81.9) & 85 (81.7) & $42(84.0)$ & 97 (82.2) & \\
\hline Presence & $40(18.1)$ & 19 (18.3) & $8(16.0)$ & $21(17.8)$ & \\
\hline Smoking status & & & & & 0.99 \\
\hline Current smoker & $71(40.6)$ & $36(44.4)$ & $17(46.7)$ & 41 (41.8) & \\
\hline Past smoker & $56(32.0)$ & 25 (30.9) & $10(26.3)$ & $32(32.7)$ & \\
\hline Non-smoker & $48(27.4)$ & $20(24.7)$ & $11(29.0)$ & 25 (25.5) & \\
\hline Drinking status & & & & & 0.53 \\
\hline Current drinker & $103(57.5)$ & $53(64.6)$ & $20(51.3)$ & $59(62.2)$ & \\
\hline Past drinker & $26(14.5)$ & $11(13.4)$ & $8(20.5)$ & 18 (18.9) & \\
\hline Non-drinker & $50(27.9)$ & $18(23.0)$ & $11(28.2)$ & 18 (18.9) & \\
\hline
\end{tabular}

Data are presented as mean \pm SD or number $(\%)$. Missing data are present.

CCRT, concurrent chemoradiotherapy; CR, complete remission; PR, partial remission; SD, stationary disease; PD, progressive disease; ECOG, Eastern Cooperative Oncology Group.

*p-values were derived from analysis of variance or chi-square test. 


\section{RESULTS}

\section{Demographic characteristics by clinical response of CCRT}

Among the 535 patients with esophageal cancer who underwent CCRT, six patients had adenocarcinoma and 36 patients had incomplete evaluation of clinical response. A total of 493 patients with ESCC completed follow-up and were evaluated for response to CCRT until July 2017 (Fig. 1). CR was higher in women than men (71\% vs 44\%) and was high in patients with good performance status (60\% in ECOG 0, 41\% in ECOG 1, 36\% in ECOG 2, and 6\% in ECOG 3 or 4). Individual underlying chronic disease, presence of other cancers, smoking status, and drinking status were not associated with CCRT response (Table 1).

\section{Tumor characteristics and completion of treatment by clinical response of CCRT}

Tumor shape, lumen involvement, length of tumor, and clinical stage were associated with CCRT response, whereas tumor pathology, chemotherapy regimen, and location had no effect on CCRT response (Table 2).

Average radiation dose and cycle of chemotherapy was similar between CR, PR, and PD, whereas radiation dose and chemotherapy cycle was significantly low in SD group (Table 2). The

Table 2. Tumor Characteristics and Treatment Completion by According to CCRT Response

\begin{tabular}{|c|c|c|c|c|c|}
\hline Characteristic & $C R(n=221)$ & PR $(n=104)$ & $\mathrm{SD}(\mathrm{n}=50)$ & $\mathrm{PD}(\mathrm{n}=118)$ & $p$-value \\
\hline Shape of tumor & & & & & $<0.001$ \\
\hline Protruding & 86 (39.8) & $32(32.0)$ & $21(41.0)$ & $32(28.6)$ & \\
\hline Ulcerative & $40(18.5)$ & $14(14.0)$ & $11(22.0)$ & 19 (17.0) & \\
\hline Combined & $66(30.6)$ & $53(53.0)$ & $18(36.0)$ & $58(51.8)$ & \\
\hline Superficial & $24(11.1)$ & $1(1.0)$ & 0 & $3(2.7)$ & \\
\hline Lumen involvement & & & & & 0.001 \\
\hline Partial & $164(74.9)$ & $64(61.5)$ & $21(42.0)$ & $64(56.1)$ & \\
\hline Circumferential & $55(25.1)$ & $40(38.4)$ & $29(58.0)$ & $50(43.9)$ & \\
\hline Location & & & & & 0.14 \\
\hline Cervical & $9(4.1)$ & $1(1.0)$ & $1(2.0)$ & $3(2.6)$ & \\
\hline Upper thorax & $32(14.5)$ & $23(22.1)$ & $4(8.0)$ & $21(18.3)$ & \\
\hline Mid thorax & $103(46.6)$ & 38 (36.5) & $25(50.0)$ & 59 (51.3) & \\
\hline Lower thorax & $77(34.8)$ & $42(40.4)$ & $20(40.0)$ & $32(27.8)$ & \\
\hline Length, cm & $4.1 \pm 2.0$ & $5.0 \pm 2.2$ & $5.1 \pm 2.8$ & $5.4 \pm 2.3$ & 0.05 \\
\hline Clinical stage & & & & & $<0.001$ \\
\hline I & $49(22.3)$ & $4(3.9)$ & $3(6.09)$ & $5(4.3)$ & \\
\hline II & 93 (42.3) & $28(27.2)$ & $11(22.0)$ & $16(13.8)$ & \\
\hline III & $52(23.6)$ & 37 (36.9) & 17 (35.3) & $41(35.3)$ & \\
\hline IV & $26(11.8)$ & 34 (33.0) & $19(38.0)$ & $54(46.6)$ & \\
\hline Radiation dose, Gy & $58.6 \pm 5.3$ & $58.9 \pm 5.0$ & $51.4 \pm 15.3$ & $57.6 \pm 9.3$ & $0.001^{\dagger}$ \\
\hline Chemotherapy cycle & $3.7 \pm 1.7$ & $3.8 \pm 1.8$ & $2.5 \pm 1.5$ & $3.5 \pm 1.9$ & $0.001^{\dagger}$ \\
\hline Incomplete CCRT & $32(14.3)$ & $41(38.7)$ & 40 (78.3) & $56(47.5)$ & 0.001 \\
\hline Cause of incomplete CCRT & & & & & $<0.001$ \\
\hline Complication/poor medical condition & $31(96.7)$ & $31(75.6)$ & $27(67.5)$ & $35(62.5)$ & \\
\hline Unresponsive (PD) & 0 & 0 & 0 & $17(30.4)$ & \\
\hline Death/refuse & $1(3.3)$ & $10(24.4)$ & $13(32.5)$ & $4(7.1)$ & \\
\hline Chemotherapy regimen & & & & & 0.19 \\
\hline FP & $191(86.4)$ & $94(90.4)$ & $40(80.0)$ & $98(83.8)$ & 0.217 \\
\hline $\mathrm{XP}$ & $24(10.9)$ & $7(6.7)$ & $10(20.0)$ & $15(12.7)$ & \\
\hline Others & $6(2.7)$ & $3(2.9)$ & 0 & $5(4.2)$ & \\
\hline
\end{tabular}

Data are presented as number (\%) or mean \pm SD. Missing data are present.

CCRT, concurrent chemoradiotherapy; CR, complete remission; PR, partial remission; SD, stationary disease; PD, progressive disease; FP, 5-fluorouracil and cisplatin; XP, capecitabine and cisplatin.

*p-values were derived from analysis of variance or chi-square test; ${ }^{\dagger}$-values were derived from t-test comparing CR and SD. 
rate of incomplete CCRT was higher in PR, SD, and PD group comparing to CR (Table 2). Main cause of incomplete CCRT was CCRT-related complication or poor medical condition in all groups $(96.7 \%$ in $\mathrm{CR}, 75.6 \%$ in $\mathrm{PR}, 67.5 \%$ in SD, and 62.5\% in PD group, respectively) and death/refuse accounted for $24 \%$ to 32\% in PR and SD group, whereas treatment discontinuation due to progressive disease accounted for 30\% in PD group (Table 2).

\section{Predicting factors of complete remission after CCRT (CR vs others)}

In univariate analysis, male sex, poor performance status ( $p$ for trend $<0.001$ ), advanced stage ( $p$ for trend $<0.001$ ), and circumferential involvement of the lumen were associated with low CR (Table 3). Protruding, ulcerative, and combined lesions were poor predictors of CR compared to superficial type.

In adjusted analysis, circumferential luminal involvement (RR, 0.61), male sex (RR, 0.31), poor performance status ( $R R$ is 0.59 in ECOG 1, 0.39 in ECOG 2, and 0.08 in ECOG 3 or 4 compared to ECOG 0), and advanced stage (RR is 0.28 in stage III and 0.12 in stage IV comparing to stage I) were poor predictors of $\mathrm{CR}$ (Table 3).

\section{Factors associated with survival and death after CCRT}

The mean survival months of patients with good performance status, good responders to CCRT, superficial tumor, and early clinical stage were long, whereas the mean survival was not different in terms of age, sex, chronic disease or other cancers, or tumor location (Supplementary Table 1). The survival rate was higher in women, those with good performance status, CR, superficial tumor, partial involvement, and early clinical stage (Supplementary Table 1).

\section{Survival analysis and Cox regression analysis}

Patients with CR had significantly high survival rate (Fig. 2A) and those with partial tumor involvement also had high survival rate (Supplementary Fig. 1A) in Kaplan-Meier curve. Patients with superficial tumor had higher survival rate than those with protruding, ulcerative, or combined types (Fig. 2B); thus, we classified patients into those with superficial tumors and other types for analysis (Supplementary Fig. 1B). Good performance status was related to high survival (Fig. $2 \mathrm{C}$ and D). Patients with stage I or II had higher survival rate than those with stage III or IV (Fig. 2E); thus, we classified the tumor stage into stage I/II and III/IV (Fig. 2F).

Table 3. Predictive Factors for Complete Remission after CCRT

\begin{tabular}{|c|c|c|c|c|}
\hline \multirow{2}{*}{ Factor } & \multicolumn{2}{|c|}{ Unadjusted analysis } & \multicolumn{2}{|c|}{ Adjusted analysis* } \\
\hline & RR (95\% CI) & $\mathrm{p}$-value & RR (95\% CI) & $\mathrm{p}$-value \\
\hline Male sex & $0.32(0.12-0.81)$ & 0.02 & $0.31(0.10-0.99)$ & 0.05 \\
\hline Age at diagnosis & $1.01(0.99-1.03)$ & 0.41 & $1.00(0.98-1.03)$ & 0.79 \\
\hline \multicolumn{5}{|l|}{ ECOG } \\
\hline 0 & 1 & & & \\
\hline 1 & $0.46(0.30-0.70)$ & $<0.001$ & $0.59(0.36-0.95)$ & 0.03 \\
\hline 2 & $0.37(0.21-0.65)$ & $<0.001$ & $0.39(0.20-0.76)$ & 0.005 \\
\hline 3 or 4 & $0.04(0.01-0.34)$ & 0.003 & $0.08(0.01-0.65)$ & 0.02 \\
\hline \multicolumn{5}{|l|}{ Shape of tumor } \\
\hline Superficial & 1 & & & \\
\hline Protruding & $0.17(0.06-0.50)$ & 0.001 & $0.35(0.09-1.32)$ & 0.13 \\
\hline Ulcerative & $0.15(0.05-0.47)$ & 0.001 & $0.36(0.09-1.44)$ & 0.15 \\
\hline Combined & $0.09(0.03-0.26)$ & $<0.001$ & $0.23(0.06-0.87)$ & 0.03 \\
\hline \multicolumn{5}{|c|}{ Lumen involvement } \\
\hline Partial & 1 & & & \\
\hline Circumferential & $0.42(0.28-0.62)$ & $<0.001$ & $0.61(0.38-0.97)$ & 0.04 \\
\hline \multicolumn{5}{|l|}{ Clinical stage } \\
\hline I & 1 & & & \\
\hline II & $0.41(0.20-0.83)$ & 0.01 & $0.61(0.27-1.39)$ & 0.24 \\
\hline III & $0.13(0.07-0.27)$ & $<0.001$ & $0.28(0.12-0.66)$ & 0.004 \\
\hline IV & $0.06(0.03-0.13)$ & $<0.001$ & $0.12(0.05-0.28)$ & $<0.001$ \\
\hline
\end{tabular}

CCRT, concurrent chemoradiotherapy; RR, relative risk; CI, confidence interval; ECOG, Eastern Cooperative Oncology Group.

*Adjusted for age, sex, ECOG, shape of tumor, lumen involvement, and clinical stage. 
A
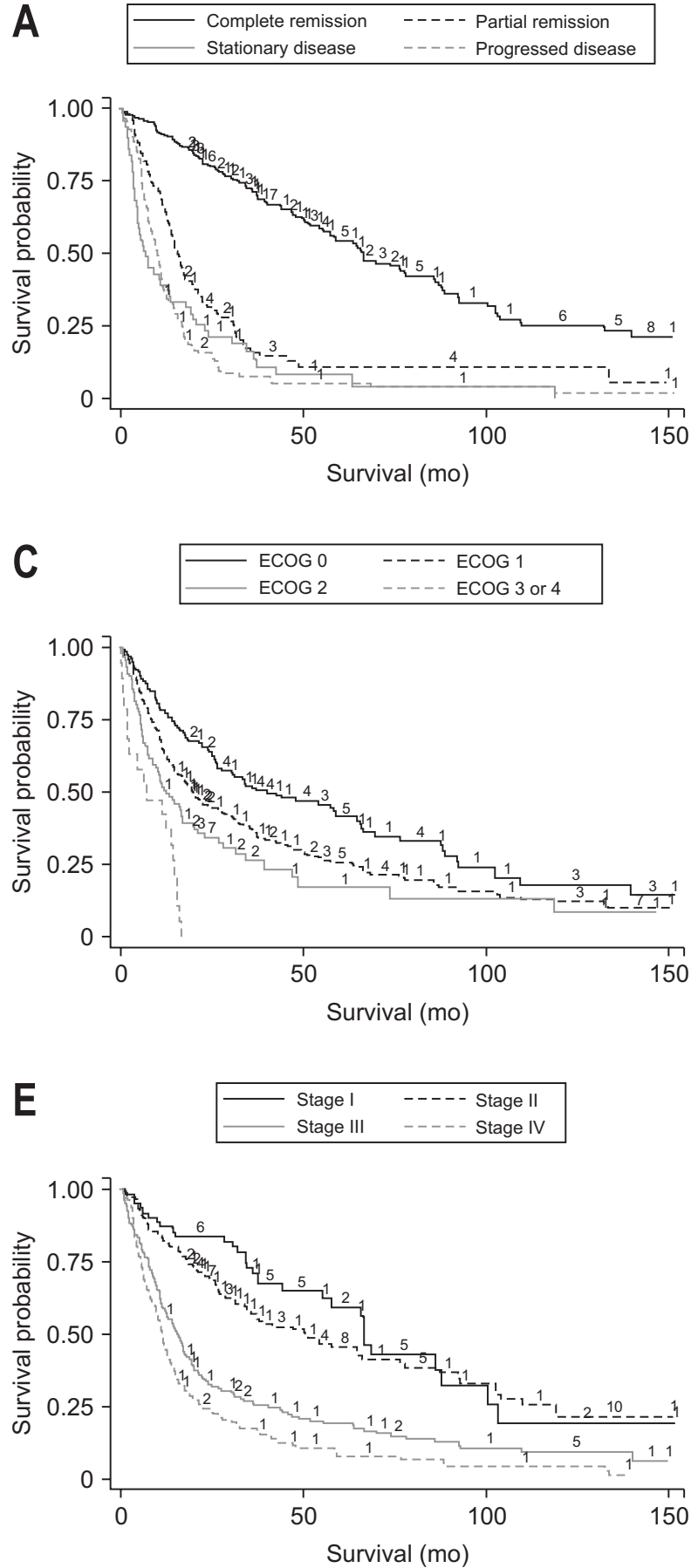
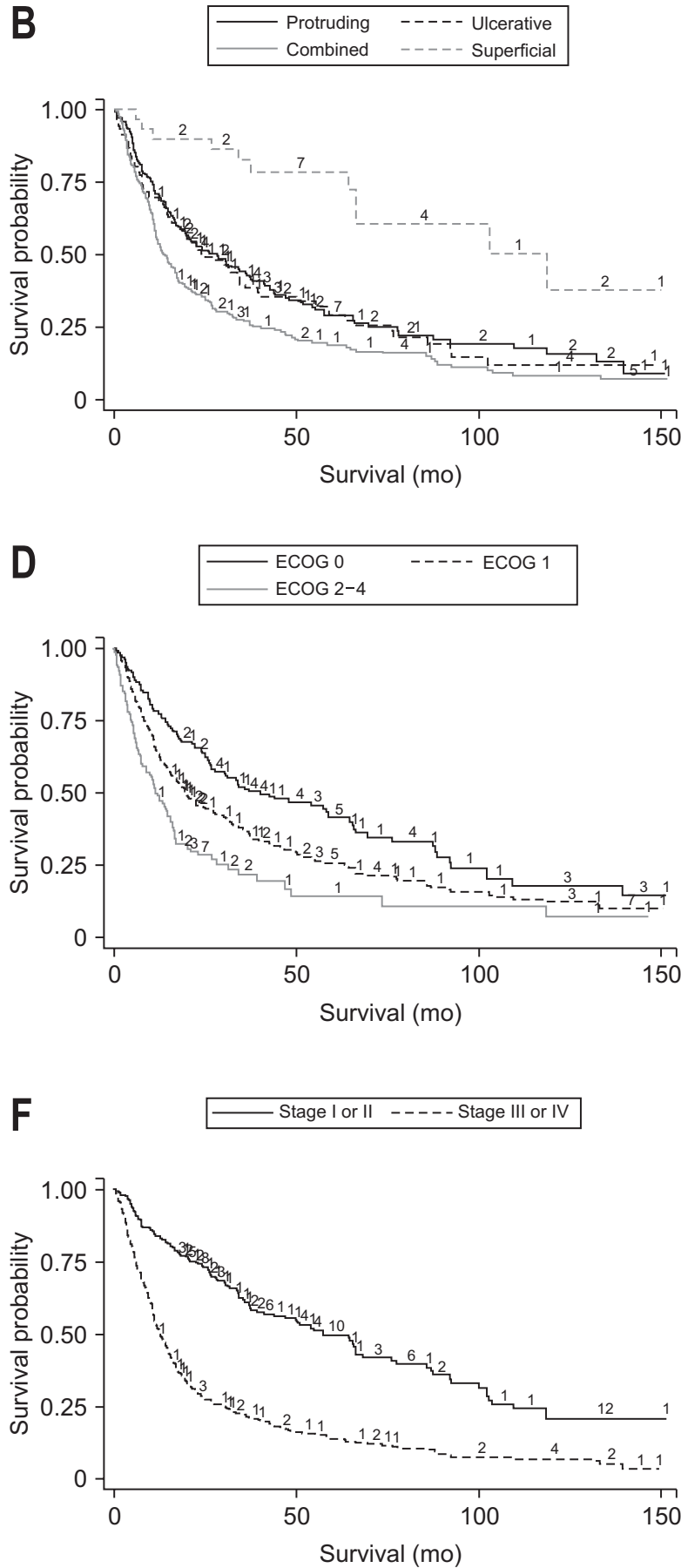

Fig. 2. Kaplan-Meier curve. (A) Survival curve according to concurrent chemoradiotherapy (CCRT) response. (B) Survival curve according to the four types of tumor shapes. (C) Survival curve according to Eastern Cooperative Oncology Group (ECOG) performance status (4 categories). (D) Survival curves according to ECOG performance status (3 categories). (E) Survival curves according to tumor stage (I, II, III, and IV). (F) Survival curves according to tumor stage (I/II and III/IV).

In adjusted analysis, poor CCRT response (HR is 2.82 in PR, 4.47 in $\mathrm{SD}$, and 4.77 in $\mathrm{PD}$ compared to $\mathrm{CR}$ ), poor performance status (HR in ECOG 2-4 is 1.38 compared to ECOG 0), and advanced stage (HR, 1.71; 95\% CI, 1.32 to 2.22 in stage I/II compared to stage III/IV) increased the mortality (Table 4). Patients with superficial tumors had lower risk of death compared to those with ulcerative or protruding tumors (HR, 0.45; 95\% CI,
0.23 to 0.90). Circumferential luminal involvement was associated with higher mortality in univariate analysis, but it had a borderline significance in adjusted analysis ( $p=0.07)$.

\section{DISCUSSION}

In the current cohort study, male sex, advanced stage, cir- 
Table 4. Predictive Factors of Mortality in Esophageal Cancer Patients Treated with CCRT

\begin{tabular}{|c|c|c|c|c|}
\hline \multirow{2}{*}{ Factor } & \multicolumn{2}{|c|}{ Unadjusted analysis } & \multicolumn{2}{|c|}{ Adjusted analysis* } \\
\hline & HR $(95 \%$ CI) & p-value & HR (95\% CI) & p-value \\
\hline Female sex & $0.74(0.43-1.26)$ & 0.26 & NA & \\
\hline Age at diagnosis & $1.00(0.98-1.02)$ & 0.94 & NA & \\
\hline \multicolumn{5}{|l|}{ Response of CCRT } \\
\hline $\mathrm{CR}$ & 1 & & 1 & \\
\hline PR & $3.57(2.68-4.75)$ & $<0.001$ & $2.82(2.08-3.81)$ & $<0.001$ \\
\hline SD & $5.42(3.83-7.67)$ & $<0.001$ & $4.47(3.12-6.41)$ & $<0.001$ \\
\hline PD & $5.68(4.31-7.47)$ & $<0.001$ & $4.77(3.52-6.47)$ & $<0.001$ \\
\hline \multicolumn{5}{|l|}{ ECOG } \\
\hline 0 & 1 & & 1 & \\
\hline 1 & $1.48(1.16-1.89)$ & 0.002 & $1.09(0.84-1.42)$ & 0.50 \\
\hline $2-4$ & $2.31(1.71-3.11)$ & $<0.001$ & $1.38(1.00-1.91)$ & 0.05 \\
\hline \multicolumn{5}{|l|}{ Shape of tumor } \\
\hline Protruding or ulcerative & 1 & & 1 & \\
\hline Superficial & $0.27(0.15-0.49)$ & $<0.001$ & $0.45(0.23-0.90)$ & 0.02 \\
\hline \multicolumn{5}{|l|}{ Lumen involvement } \\
\hline Partial & 1 & & 1 & \\
\hline Circumferential & $1.68(1.37-2.07)$ & $<0.001$ & $1.23(0.98-1.54)$ & 0.07 \\
\hline \multicolumn{5}{|l|}{ Clinical stage } \\
\hline I or II & 1 & & 1 & \\
\hline III or IV & $2.79(2.24-3.48)$ & $<0.001$ & $1.71(1.32-2.22)$ & $<0.001$ \\
\hline
\end{tabular}

CCRT, concurrent chemoradiotherapy; HR, hazard ratio; CI, confidence interval; NA, not available; CR, complete remission; PR, partial remission; SD, stationary disease; PD, progressive disease; ECOG, Eastern Cooperative Oncology Group.

*Adjusted for CCRT response, ECOG, shape of tumor, lumen involvement, and clinical stage.

cumferential luminal involvement, and poor performance status (high ECOG) were strong predicting factors for poor CCRT response. Advanced stage, poor CCRT response, and poor performance status increased mortality, whereas superficial tumor reduced mortality compared to ulcerative or protruding tumors.

In the current study, advanced stage was the strong predictor of poor CCRT response. CCRT achieved CR in $80 \%$ of patients with stage I, 63\% of patients with stage II, 35\% of patients with stage III, and 19\% of patients with stage IV. In adjusted analysis, the RR of CR was 0.28 in stage III and 0.12 in stage IV compared to stage I. In previous studies, CCRT lead to CR in 15\% to $33 \%$ of patients with T4 ESCC. ${ }^{10}$ A total of $65 \%$ of patients with stage II or III ESCC who underwent CCRT, achieved CR. ${ }^{11}$ Although definitive CCRT is a therapeutic strategy for locally advanced esophageal cancer, the incidence of recurrence after $\mathrm{CR}$ is frequent, and some patients do not achieve remission with CCRT alone. Previous studies suggested that clinical stage predicted the response to CCRT. ${ }^{5-7}$

In adjusted analysis, good performance status was an independent predictor of CR with RR of 0.59 in ECOG 1, 0.39 in ECOG 2, and 0.08 in ECOG 3 or 4 compared to ECOG 0. In a single-center study (142 patients with stage III and IV ESCC receiving CCRT), predictors of CCRT responsiveness were Glasgow
Prognostic scores, ECOG performance status, and cTNM stage. ${ }^{12}$ Patients with good ECOG performance status tolerate CCRT; thus, they can receive full-dose chemoradiation and complete the chemotherapy cycle. Actually, completion of CCRT was significantly high in CR group (85.7\%) than other groups (21.7\% to $61.3 \%$ ). Therefore, good performance status seems to be a reliable predictor of $\mathrm{CR}$.

CR was higher in women than in men (71\% vs $44 \%)$. In adjusted analysis, male sex was a predicting factor for poor CCRT response, and hormonal difference may attribute to different response between men and women. In esophageal cancer, sex differences in CCRT response have not been noted. The incidence of esophageal cancer is low in women; therefore, measuring sex difference in CCRT response can have statistical error. A large data analysis or meta-analysis and combined molecular study may clarify this difference.

In this study, circumferential luminal involvement was another independent predictor for poor CCRT response. Patients with circumferential involvement tend to have advanced $\mathrm{T}$ stage and high probability to develop comorbidities and stricture during CCRT, resulting in a high rate of CCRT discontinuation. Although superficial tumor produced higher CR than protruding or infiltrative type in univariate analysis, its effect was ameliorated 
in adjusted analysis. Superficial and non-superficial types are strongly related with $\mathrm{T}$ stage, and therefore its effect on CCRT response appears to be eliminated in adjusted analysis. CCRT response in terms of tumor pathology and location was not different. Individual underlying chronic disease, presence of other cancers, smoking status, and drinking status had no association with CCRT response.

Only CR patients can achieve significantly better survival than other groups. This is the reason we have to predict probability of CR in patients receive CCRT. In adjusted analysis, poor CCRT response, advanced stage (stage III/IV), and poor ECOG performance status independently increased the risk of death, whereas superficial tumor reduced the risk of death. In a previous study that included 116 patients, survival was associated with clinical CR, less weight loss, and good World Health Organization performance. ${ }^{13}$ Tumor persistence and recurrence as well as distant metastasis are significant factors for death in advanced cancer. A previous meta-analysis suggested that CCRT controlled local tumor and prevented recurrence; there was no difference between patients treated with CCRT and RT alone in the aspect of distant metastasis. ${ }^{14}$ In a previous study, considerable esophageal luminal stenosis was associated with a higher T stage and longer tumor length and it was related with poor overall survival in ESCC. ${ }^{15}$ In our study, we adjusted for associated factors with luminal stenosis such as stage, degree of lumen involvement (circumferential vs partial), and tumor shape (superficial vs protruding/ulcerative). In unadjusted analysis, they were all strongly associated with survival, whereas advanced stage and tumor shape remained strong contributing factors on mortality and degree of lumen involvement had a borderline significance in adjusted analysis.

This study has several strengths. First, this was a large study that evaluated the predictive factors of CCRT responsiveness for esophageal cancer. Thus, we performed a stratified and adjusted analysis to investigate predicting factors for CCRT responsiveness and survival. Second, we evaluated the rate of death or survival using both hospital data and data from the National Health Insurance System. When individuals die, their death is recorded in the National Health Insurance System. Therefore, we were able to minimize follow-up loss and selection bias by follow-up loss. Nevertheless, it has some limitations. First, this is a retrospective cohort study. Therefore, chronic disease and status of smoking and drinking may be less exact comparing to prospective studies. Second, the clinical parameters have limitation to predict CCRT response and survival. Emerging research on biomarkers may provide more accurate prediction of outcomes with CCRT. Several biomarkers were suggested to have association with CCRT response such as ALDH- $1,{ }^{16} \mathrm{NF}-\kappa \mathrm{B},{ }^{17} \mathrm{p} 53,{ }^{18}$ BRCA $1,{ }^{19}$ and ERCC $1 .{ }^{20}$ Some genetic profile may provide prediction of CCRT responsiveness. ${ }^{21,22}$ Expression of miRNA may predict the CCRT response. ${ }^{23,24}$ The candidate biomarkers should be validated in large population to use for clinical application.
Third, we did not analyze definite CCRT and palliative CCRT separately due to limited sample size. Last, we did not perform external validation.

In conclusion, women, good performance status, early stage, and superficial tumors are independent predicting factors for CR after CCRT in esophageal cancer. Advanced stage, poor CCRT response, and poor performance status independently increased the risk of cancer death, whereas superficial tumor reduced the death risk.

\section{CONFLICTS OF INTEREST}

No potential conflict of interest relevant to this article was reported.

\section{ACKNOWLEDGEMENTS}

This study was funded by the National Research Foundation of Korea (NRF-2015R1D1A1A01059219).

\section{AUTHOR CONTRIBUTIONS}

Study concept and design: S.W.J., S.Y.N. Data acquisition: S.W.J., S.Y.N., Y.H.K., H.S.L., S.J.L., S.K.K. Data analysis and interpretation: S.Y.N., S.W.J. Drafting of the manuscript: S.Y.N. S.J.L., S.W.J. Critical revision of the manuscript for important intellectual content: S.Y.N., Y.H.K., H.S.L., S.K.K., S.W.J. Statistical analysis: S.Y.N. Obtained funding: S.Y.N. Administrative, technical, or material support; study supervision: S.W.J. Approval of final manuscript: all authors.

\section{ORCID}

Su Youn Nam https://orcid.org/0000-0002-5568-7714

Seong Woo Jeon $\quad$ https://orcid.org/0000-0002-9539-9389

Sang Jik Lee https://orcid.org/0000-0001-8627-3509

Yong Hwan Kwon https://orcid.org/0000-0002-0520-9685

Hyun Seok Lee

https://orcid.org/0000-0001-9288-0321

Sung Kook Kim

https://orcid.org/0000-0002-2861-8123

\section{REFERENCES}

1. Bray F, Ferlay J, Soerjomataram I, Siegel RL, Torre LA, Jemal A. Global cancer statistics 2018: GLOBOCAN estimates of incidence and mortality worldwide for 36 cancers in 185 countries. CA Cancer J Clin 2018;68:394-424.

2. Pickens A, Orringer MB. Geographical distribution and racial disparity in esophageal cancer. Ann Thorac Surg 2003;76:S1367S1369.

3. Siegel RL, Miller KD, Jemal A. Cancer statistics, 2016. CA Cancer J Clin 2016;66:7-30.

4. al-Sarraf M, Martz K, Herskovic A, et al. Progress report of 
combined chemoradiotherapy versus radiotherapy alone in patients with esophageal cancer: an intergroup study. J Clin Oncol 1997;15:277-284.

5. Amini A, Ajani J, Komaki R, et al. Factors associated with localregional failure after definitive chemoradiation for locally advanced esophageal cancer. Ann Surg Oncol 2014;21:306-314.

6. Nomura M, Shitara K, Kodaira T, et al. Recursive partitioning analysis for new classification of patients with esophageal cancer treated by chemoradiotherapy. Int J Radiat Oncol Biol Phys 2012;84:786-792.

7. Chao YK, Tseng CK, Wen YW, et al. Using pretreatment tumor depth and length to select esophageal squamous cell carcinoma patients for nonoperative treatment after neoadjuvant chemoradiotherapy. Ann Surg Oncol 2013;20:3000-3008.

8. Bedenne L, Michel P, Bouché 0 , et al. Chemoradiation followed by surgery compared with chemoradiation alone in squamous cancer of the esophagus: FFCD 9102. J Clin Oncol 2007;25:1160-1168.

9. Stahl M, Stuschke M, Lehmann N, et al. Chemoradiation with and without surgery in patients with locally advanced squamous cell carcinoma of the esophagus. J Clin Oncol 2005;23:2310-2317.

10. Ishida K, Ando N, Yamamoto S, Ide H, Shinoda M. Phase II study of cisplatin and 5-fluorouracil with concurrent radiotherapy in advanced squamous cell carcinoma of the esophagus: a Japan Esophageal Oncology Group (JEOG)/Japan Clinical Oncology Group trial (JCOG9516). Jpn J Clin Oncol 2004;34:615-619.

11. Kato K, Muro K, Minashi K, et al. Phase II study of chemoradiotherapy with 5-fluorouracil and cisplatin for stage II-III esophageal squamous cell carcinoma: JCOG trial (JCOG 9906). Int J Radiat Oncol Biol Phys 2011;81:684-690.

12. Kimura J, Kunisaki C, Makino H, et al. Evaluation of the Glasgow Prognostic Score in patients receiving chemoradiotherapy for stage III and IV esophageal cancer. Dis Esophagus 2016;29:10711080.

13. Di Fiore F, Lecleire S, Rigal O, et al. Predictive factors of survival in patients treated with definitive chemoradiotherapy for squamous cell esophageal carcinoma. World J Gastroenterol 2006;12:41854190.

14. Zhu LL, Yuan L, Wang H, et al. A meta-analysis of concurrent chemoradiotherapy for advanced esophageal cancer. PLoS One 2015;10:e0128616.

15. Yang YS, Hu WP, Ni PZ, Wang WP, Yuan Y, Chen LQ. Esophageal luminal stenosis is an independent prognostic factor in esophageal squamous cell carcinoma. Oncotarget 2017;8:43397-43405.

16. Ajani JA, Wang X, Song S, et al. ALDH-1 expression levels predict response or resistance to preoperative chemoradiation in resectable esophageal cancer patients. Mol Oncol 2014;8:142-149.

17. Izzo JG, Malhotra U, Wu TT, et al. Association of activated transcription factor nuclear factor kappab with chemoradiation resistance and poor outcome in esophageal carcinoma. J Clin Oncol 2006;24:748-754.

18. Makino T, Yamasaki M, Miyata H, et al. p53 mutation status predicts pathological response to chemoradiotherapy in locally advanced esophageal cancer. Ann Surg Oncol 2010;17:804-811.

19. Gao Y, Zhu J, Zhang X, et al. BRCA1 mRNA expression as a predictive and prognostic marker in advanced esophageal squamous cell carcinoma treated with cisplatin- or docetaxel-based chemotherapy/chemoradiotherapy. PLoS One 2013;8:e52589.

20. Brabender J, Vallböhmer D, Grimminger P, et al. ERCC1 RNA expression in peripheral blood predicts minor histopathological response to neoadjuvant radio-chemotherapy in patients with locally advanced cancer of the esophagus. J Gastrointest Surg 2008;12:1815-1821.

21. Duong C, Greenawalt DM, Kowalczyk A, et al. Pretreatment gene expression profiles can be used to predict response to neoadjuvant chemoradiotherapy in esophageal cancer. Ann Surg Oncol 2007;14:3602-3609.

22. Wen J, Yang H, Liu MZ, et al. Gene expression analysis of pretreatment biopsies predicts the pathological response of esophageal squamous cell carcinomas to neo-chemoradiotherapy. Ann Oncol 2014;25:1769-1774.

23. Wen J, Luo K, Liu H, et al. MiRNA expression analysis of pretreatment biopsies predicts the pathological response of esophagea squamous cell carcinomas to neoadjuvant chemoradiotherapy. Ann Surg 2016;263:942-948.

24. Skinner HD, Lee JH, Bhutani MS, et al. A validated miRNA profile predicts response to therapy in esophageal adenocarcinoma. Cancer 2014;120:3635-3641. 